\title{
MAPEO DE ZONAS VULNERABLES A DESLIZAMIENTOS USANDO PP GIS Y TÉCNICAS DE TELEDETECCIÓN
}

\author{
MAPPING OF VULNERABLE ZONES TO SLIDES USING PP GIS AND \\ TELEDETECTION TECHNIQUES
}

NATALIA PACURUCU CACERES ${ }^{1}$, ENRIQUE ACOSTA² VILLIE MOROCHO ${ }^{3}$

${ }^{1}$ FACULTAD DE ARQUITECTURA Y URBANISMO.

${ }^{2}$ FACULTAD DE INGENIERIA

${ }^{3}$ DEPARTAMENTO DE CIENCIAS DE LA COMPUTACIÓN. FACULTAD DE INGENIERÍA.

Universidad de Cuenca. Avenida 12 de Abril y Agustín Cueva, Cuenca, Ecuador

natalia.pacurucu@ucuenca.edu.ec; villie.morocho@ucuenca.edu.ec; enrique.acosta@ucuenca.edu.ec

Recibido: 16 de agosto de 2018 / Aceptado: 27 de noviembre de 2018

\section{RESUMEN}

Las nuevas tecnologías de la información y la comunicación permiten que no solo los técnicos especializados sean los que generen información geoespacial. En la actualidad sistemas como los PP GIS, permiten que la población pueda ser generadora de información, permitiendo así que los datos geoespaciales puedan ser actualizados constantemente. En este artículo se presenta una aplicación PP GIS que ha permitido levantar datos geoespaciales de zonas vulnerables a deslizamientos en el cantón Cuenca y se ha comparado con la información levantada a través de técnicas de teledetección como son los drones y el láser scanner terrestre.

Palabras clave: participación ciudadana; NTIC; PP GIS; datos geoespaciales; zonas vulnerables; deslizamientos.

\begin{abstract}
The new information and communication technologies allow not only specialized technicians to generate geospatial information. At present, systems such as PP GIS allow the population to generate information, thus allowing geospatial data to be constantly updated. This article presents a PP GIS application that has allowed to collect geospatial data from vulnerable areas to landslides in the canton of Cuenca and has been compared with the information raised through remote sensing techniques such as drones and the terrestrial laser scanner.
\end{abstract}

Key words: public participation; NTIC; PPGIS; geospatial date; vulnerable áreas; landslides

\section{INTRODUCCIÓN}

Este trabajo propone la identificación de zonas vulnerables a través del uso de las NTIC en conjunto con la participación ciudadana, de manera que esta información sirva de insumo para los procesos de ordenación territorial, debido a que el Ecuador regularmente enfrenta las catástrofes de eventos naturales y antrópicos que afectan severamente a la población, las actividades productivas y las inversiones (Morocho, 2013). 
Para la planificación territorial es necesario que la cartografía esté lo más actualizada posible. En el caso de las zonas vulnerables más aún se debe contar con mapas con información fiable de manera que permita la toma de decisiones oportuna, ya sea frente a un desastre o para la planificación del uso del suelo, tomando en cuenta que en este tipo de áreas en nuestro país se encuentren asentamientos poblacionales.

En esta investigación se ha generado una aplicación PPGIS que ha sido integrada a la IDE Ucuenca y se ha probado el uso de la misma en tres zonas de estudio del cantón Cuenca. Los datos proporcionados por la población a través de la aplicación móvil han sido comparados con los datos geoespaciales obtenidos de la aplicación de laser scanner terrestre y vuelos aéreos de un drone.

\section{IDENTIFICACIÓN DE LAS ZONAS DE ESTUDIO}

Según el Plan de Ordenamiento Territorial de la Provincia del Azuay, el territorio presenta varias áreas de afección con riesgo muy alto, debido a amenazas latentes como es el caso de deslizamientos derrumbes, o caídas de rocas, que ponen en peligro a sectores con presencia de infraestructura y/o población, convirtiendo el área en una zona de alto riesgo inminente para la vida de los habitantes como para el transporte y comunicación en el caso de las vías (GAD Azuay, 2015).

Dentro de la provincia del Azuay, en el cantón Cuenca, presenta mayor frecuencia de deslizamientos. Los sectores de Nulti, Los Trigales, Llacao, Paccha, Turi, Sinincay, Sayausí y Sidcay son los sectores que durante muchos años soportan este problema. Si bien durante algún tiempo los deslizamientos bajaron su intensidad, eso no significó que el peligro pasó (El Tiempo, 2010).

En la Figura 1 se observan las amenazas a nivel del Azuay, en color rojo se presentan los deslizamientos, evidenciándose más en las zonas de Santa Isabel y San Fernando; en tanto que para el cantón Cuenca es más notable las zonas con amenazas a material aluvial, reptación, hundimientos y asentamientos.

De acuerdo a la información anterior, el cantón Cuenca se ha seleccionado como una zona macro para el estudio. Para la identificación de las zonas especificas se recopilo información de noticias a nivel local en las que se hayan registrado diferentes sucesos respecto a deslizamientos. Con esta información se conformó una matriz, en la que se constató la frecuencia de los eventos para las distintas localidades, encontrando que se ha repetido mayormente estos sucesos en Los Trigales y Reina del Cisne y otra zona en la que el nivel de afección ha sido alto, tal es el caso de Chalacay. Cabe destacar que durante el proceso se descartó Chalacay por inconvenientes como el clima de la zona para los levantamientos de información, daños en los equipos y dificultades relacionadas con el desplazamiento pues la zona se encuentra a una distancia de $75 \mathrm{Km}$. de la ciudad de Cuenca. Por ello, se ha buscado un lugar en el que las infraestructuras presentes estén 
siendo afectadas por algún otro deslizamiento. Así se ha identificado el deslizamiento del sector de la 24 de Mayo - Universidad del Azuay - autopista Cuenca-Azogues. Estos tres asentamientos han sido entonces seleccionados como Áreas Específicas de Estudio, a continuación se describe cada una de ellas.

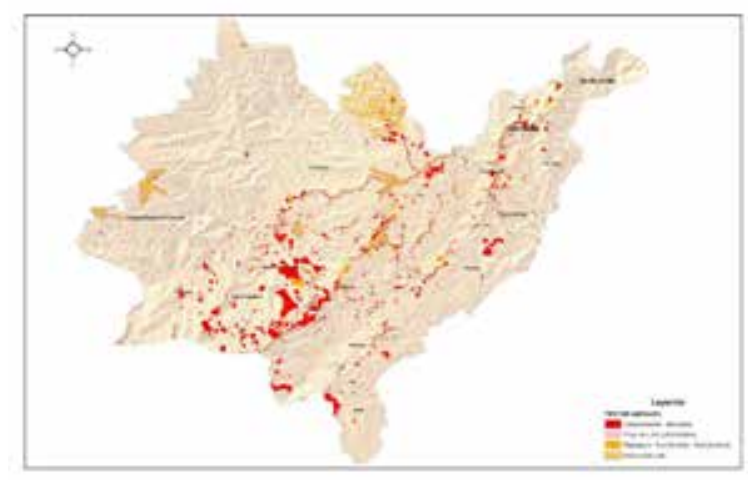

Figura 1. Movimientos de masa de suelo de la provincia del Azuay.

Fuente: estudios e investigación de amenaza, vulnerabilidad y riesgos 2012, unidad de riesgos del g.P.A. Elaboración: Dirección de Planificación G.P.A.

\section{TRIGALES ALTOS}

Se encuentra ubicada al Noreste del área urbana del cantón Cuenca, en la parroquia Hermano Miguel.

En el 2007 el movimiento de tierra afectó, por primera vez, a los habitantes de Trigales Altos. La administración municipal de aquel entonces realizó pequeños desfogues para el agua. Con ello, el movimiento frenó, pero luego de dos años, a finales del 2009, nuevamente los deslizamientos afectaron al sector. En total 105 edificaciones resultaron afectadas con paredes cuarteadas y pisos rotos; se lograron desalojar a varias familias y en las fachadas de las casas se pintó el mensaje: "Zona en Riesgo" (El Tiempo, 2010). El límite del área de estudio está entre las calles De La Liclla, Coronel Monsalve y Gregorio Cordero Crespo (Ver Figura 2).

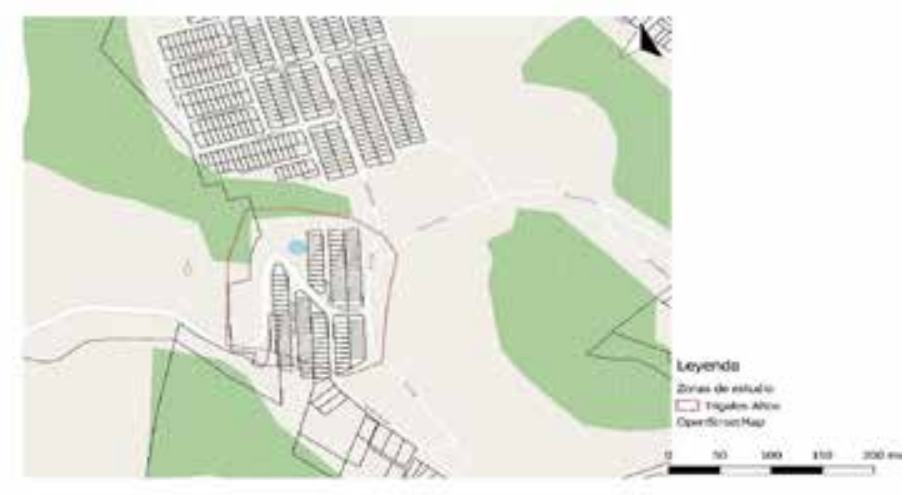

Figura 2. Limite de la zona de estudio en Trigales Altos.

Fuente: Elaboración Propia 


\section{REINA DEL CISNE}

Es una comunidad de la parroquia Paccha, localizada al Sureste del cantón Cuenca.

Paccha es una parroquia rural en donde aún se mantiene la población indígena. La población -situada en las faldas del Guagualluma, en terrenos muy deleznables- era indígena que ya existía antes de la conquista, y fue fundada por los españoles en el año 1582 con el nombre de San Francisco de Paccha, integrando de inmediato el antiguo Corregimiento de Cuenca.

A partir de 1957, una serie de deslizamientos advirtieron a la población que sus condiciones geológicas eran peligrosas; estos deslizamientos cobraron mayor intensidad a partir de 1993 y obligaron a muchos de sus pobladores a abandonar sus casas, muchas de ellas destruidas o «tragadas» por la tierra («Paccha (Azuay) - Geografía del Ecuador», 2016).

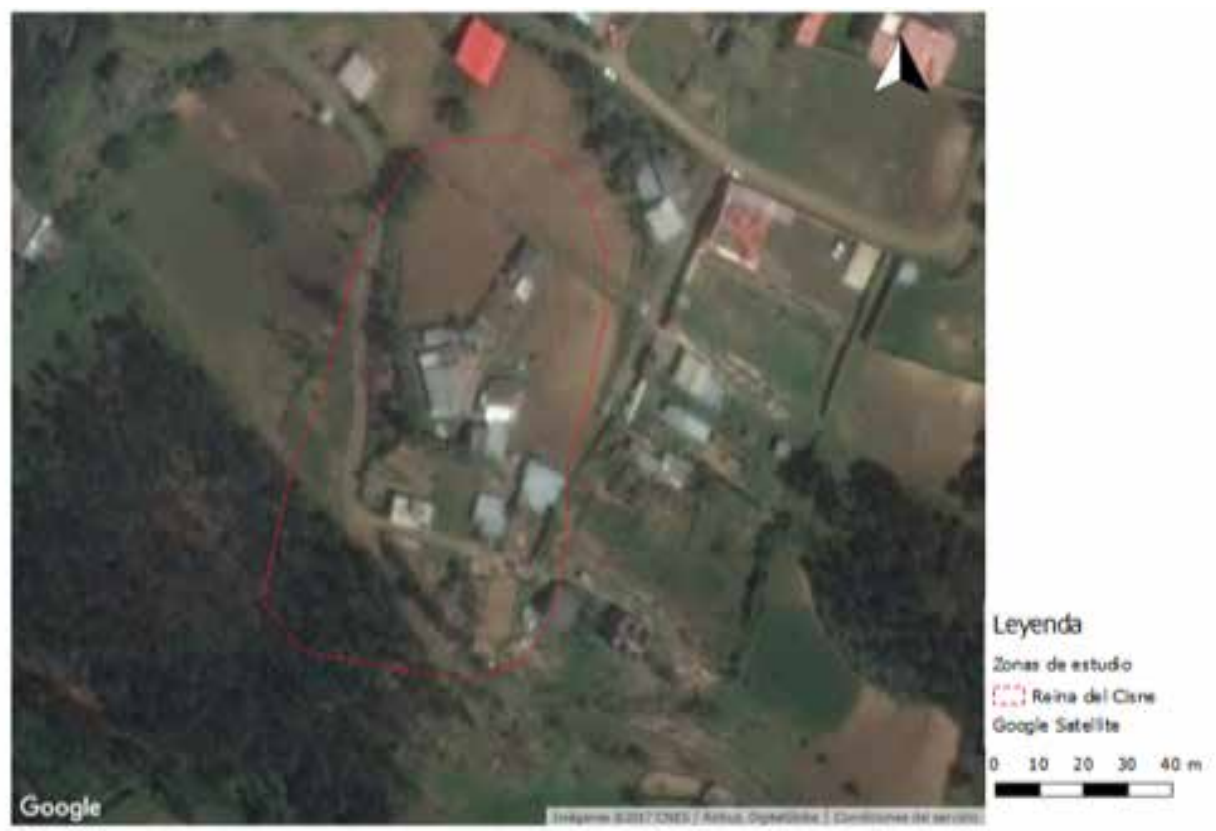

Figura 3. Límite de la zona de estudio en Reina del Cisne.

Fuente: Elaboración Propia

\section{SECTOR GAPAL-UNIVERSIDAD DEL AZUAY}

La zona de estudio se encuentra localizada en la ciudad de Cuenca, cabecera cantonal del cantón del mismo nombre. El sector está ubicado al Surde la ciudad limitado por la Av. 24 de Mayo, la Circunvalación Sur y parte de la Parroquia Turi. Es el más grande de los tres deslizamientos estudiados. La mayor parte del deslizamiento afecta a un depósito coluvial sobre el que también encontramos la autopista Cuenca-Azogues, parte del campus universitario de la UDA y una zona residencial situada junto a la Av. 24 de mayo. 


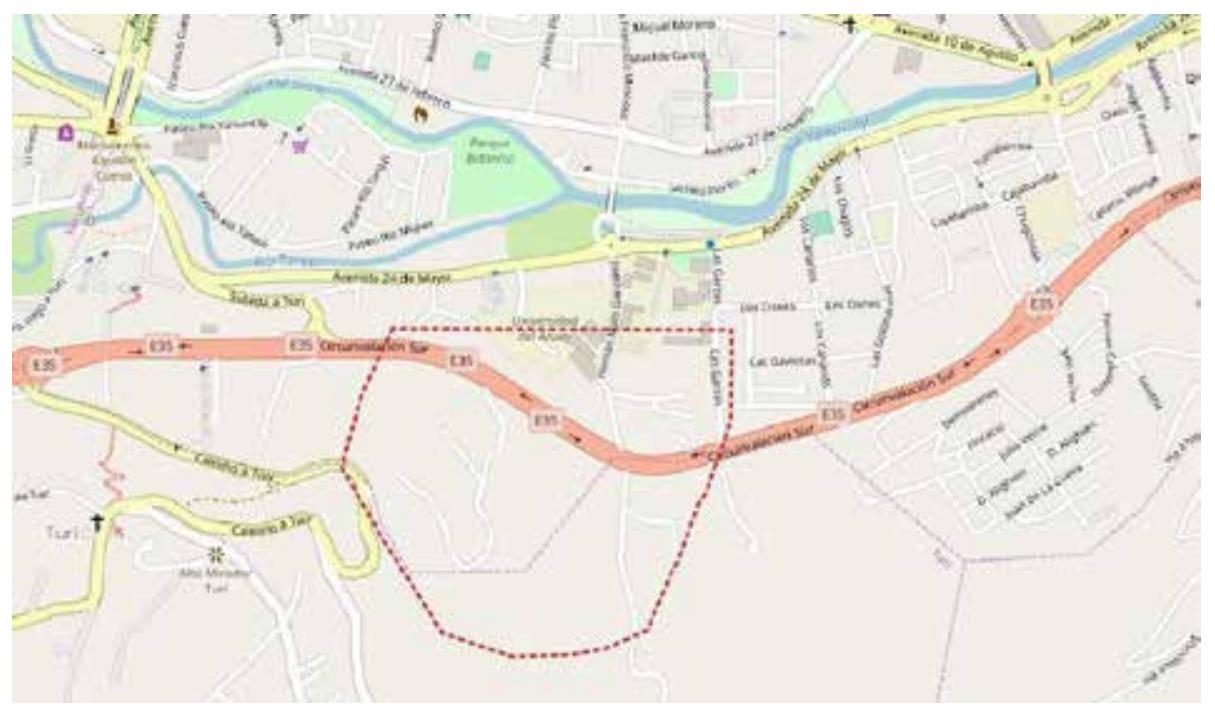

Figura 4. Localización zona de estudio sector UDA.

Fuente: Elaboración Propia

El sector comenzó a presentar problemas de hundimientos a partir de la construcción de la Circunvalación Sur. Según técnicos, el movimiento de tierra activó una falla geológica que estuvo a punto de derribar las edificaciones de la UDA (El Tiempo, 2012).

No solo las edificaciones de la Universidad del Azuay han sufrido estragos, varios barrios cercad de la zona como el Cinco de Junio, El Romero han quedado afectados, algunas viviendas han tenido que ser deshabitadas ante el peligro que se desplomen («Drama en el Azuay por deslizamientos», 2011).

En la zona se presentaban quebradas de drenaje natural, sin embargo al construir la autopista se rellenaron y no se colocaron los drenajes adecuados, provocando cambios de régimen en nivel freático (Diario El Mercurio, 2011).

\section{MAPEO DE DESLIZAMIENTOS EN LAS ZONAS DE ESTUDIO A TRAVÉS DE TÉCNICAS DE TELEDETECCIÓN Y PARTICIPACIÓN CIUDADANA}

\section{TÉCNICAS DE TELEDETECCIÓN USADAS}

Como técnicas de teledetección se ha utilizado los vuelos a través de un vehículo aéreo no tripulado y el uso de laser scanner terrestre. Los datos obtenidos fueron pos procesados generando así la cartografía actualizada de los deslizameientos de las tres zonas de estudio. A continuación se describen los materiales utilizados en el proceso:

- Escáner láser terrestre (TLS) FARO Focus 3D X130 (Figura 15A). 
- Drone multirotor DJI Phantom 4 equipado con cámara RGB 4K, propiedad del Ing. Gerardo Arbito (Figura 15B).

- Drone de ala fija Sensefly eBee AG equipado con cámara RGB (Sony S110) y multiespectral de 5 bandas (Sensefly Sequoia) (Figura 15C).

- Estación total Ruide (Figura 15D).

- Estación total Trimble R3 (Figura 15E).

- GPS diferencial Trimble R4 (Figura 15F).

- Equipo de tomografía de resistividad eléctrica (ERT) y tomografía sísmica de refracción PASI 16S24-N del Ing. Alfredo Vázquez (Figura 15G).

- Equipo de tomografía de resistividad eléctrica (ERT) de la Red Sísmica del Austro (RSA) IRIS Instruments SYSCAL Pro. SWITHC 48 (Figura 15H).

- Fotografías aéreas del IGM de los años 60, 70, 80, 90 y 2000 y estereoscopio (Figura 15I).

- Brújula para medición de estructuras geológicas (Figura 15J).

- Tamices para granulometría (Figura 15K).

- Útiles de laboratorio para determinar densidad de las muestras de suelo (Figura 15L).

- Cuchara de Casagrande para el límite líquido (Figura 15M).

- Edómetro para realizar el ensayo de expansividad de arcillas (Figura 15N).

Para el mapeo de las zonas, se recopiló información existente, fundamentalmente en el Departamento de Geomática del Municipio de Cuenca y en la oficina de Gestión del Riesgo.

Se tomaron muestras de suelo en campo y realización de ensayos geotécnicos en laboratorio de suelos de la Universidad de Cuenca para determinar ciertas propiedades físicas y mecánicas de dichas muestras.

Ya en campo, se realizaron levantamientos parciales del deslizamiento (a lo largo del perfil longitudinal) con TLS (escáner láser terrestre) en distintas fechas y se realizó la unión de los escaneos individuales de cada fecha con Scene o AutoDesk Recap 360.

Los primeros resultados de las nubes de puntos TLS de distintas fechas fueron comparados a partir de 3 puntos de referencia externos al deslizamiento. Esta tarea se ha realizado con CloudCompare.

Posterior a este proceso se generó los perfiles longitudinales y transversales en AutoCAD Civil 3D para la acotación de los movimientos que ha experimentado el terreno y las infraestructuras situadas sobre él, validando los resultados obtenidos con TLS mediante puntos de control medidos con GPS diferencial y estación total.

El levantamiento fotogramétrico multitemporal del deslizamiento completo se obtuvo mediante un drone equipado con cámara RGB y multiespectral. La precisión de estos levantamientos es menor que la del TLS. Sin embargo, la fotogrametría 
drone proporciona una valiosa información más allá del perfil longitudinal del deslizamiento, donde no llega el TLS.

Una vez realizado el vuelo, se obtuvo la nube de puntos, modelo digital de superficie (MDS), modelo digital del terreno (MDT), ortofoto y modelos de índice de vegetación (NDVI) mediante Pix4D, Agisoft Photoscan y QGIS.

Estas nubes de puntos fotogramétricas de distintas fechas fueron comparadas con CloudCompare a partir de puntos de referencia estáticos situados fuera del deslizamiento. Los resultados se validaron mediante puntos de control medidos con GPS diferencial y estación total.

Finalmente se realizaron prospecciones geofísicas con equipos de tomografía de resistividad eléctrica (ERT) y tomografía sísmica para identificar la morfología y profundidad de la superficie del deslizamiento, y por lo tanto la tipología del mismo.

\section{APLICACIÓN PP GIS PARA EL LEVANTAMIENTO DE INFORMACIÓN}

Los PP GIS son sistemas que permiten la participación ciudadana, a través de la recopilación de información cartográfica relevante que pueden convertirse en insumos para la planificación territorial. Con estos sistemas la población podrá no solo proporcionar datos, sino también capacitarse y concientizarse sobre cómo usar el suelo y con ello evitar los asentamientos en zonas vulnerables.

En esta investigación se ha generado una aplicación de participación ciudadana SIG en la que la población puede proporcionar datos específicos sobre zonas que ya han sufrido deslizamientos o propensas a este fenómeno de manera que los investigadores o los tomadores de decisiones puedan contar con datos actualizados y geo localizados de estas zonas.

Para receptar la información proveniente de los ciudadanos tanto que residen en zonas propensas a deslizamientos como aquellos que no, se ha generado una aplicación web móvil con la que la población puede alertar sobre estos fenómenos y localizar las áreas afectadas. Con estos insumos se podrán generar mapas más precisos y que evitarán futuros desastres como asentamientos, aportando así a la planificación territorial.

La App móvil "PPGIS UC" fue desarrollada en la Plataforma Android Studio, con la finalidad de realizar reportes de eventos de vulnerabilidad, mediante la selección y registro de ciertos parámetros en formularios dinámicos. En el proyecto que se ejecutó para el desarrollo de esta aplicación, se centró en el reporte para deslizamientos de terrenos, pero, la aplicación está diseñada para adaptarse a diferentes escenarios. 


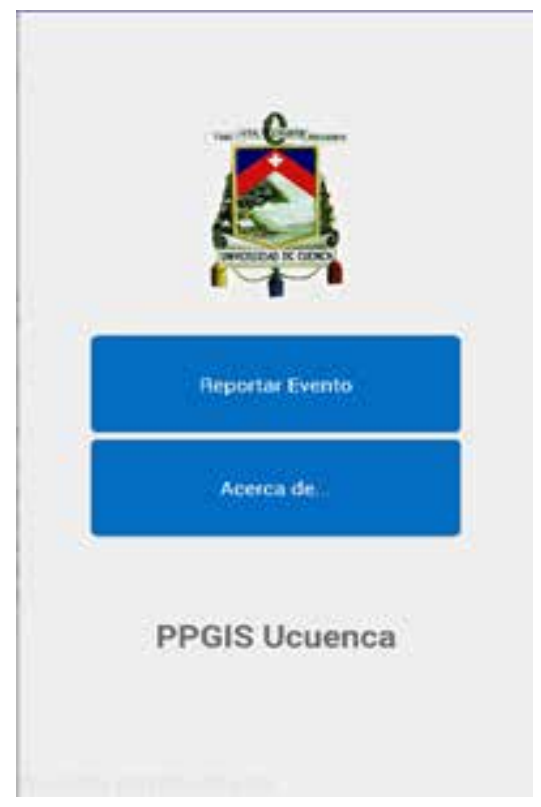

Figura 5. Interfaz de la aplicación

Los reportes que se realizan son enviados a la Plataforma IDE y son:

- Tabla que registra todos los reportes realizados.

- Clasificación de la Información.

- Generación de geometrías y almacenamiento en la Base de Datos espacial PostGIS.

- Publicación en el servidor de mapas GeoServer (Es posible utilizar los diferentes servicios WMS, WFS para utilizarlos en diferentes herramientas GIS).

- Visualización de la Información en un Visualizador de Mapas.

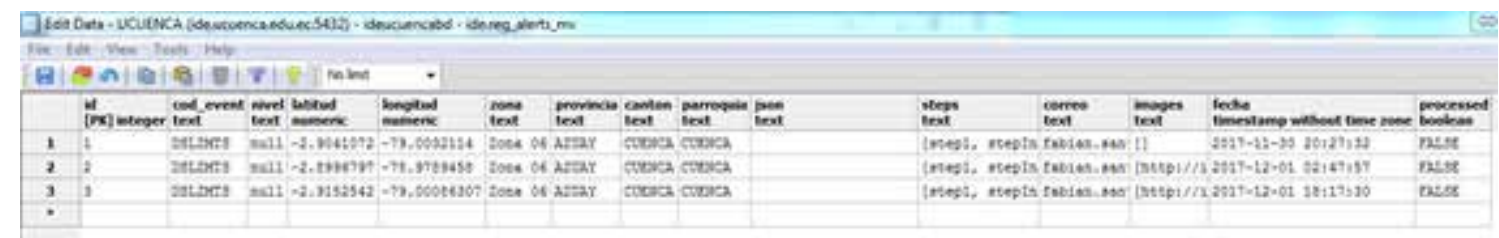

Figura 6. Base de datos de almacenamiento

La aplicación permite reportar eventos sin conexión a internet, y posteriormente ser enviada la información al servidor al detectar una conexión a internet de manera automática.

Tiene la posibilidad de poner a disposición del usuario varios tipos de formularios (por ejemplo, para un ciudadano común o experto); estos formularios pueden ser integrados o removidos desde la plataforma IDE de la Universidad de Cuenca. 


\section{ANÁLISIS DE LAS ZONAS DE ESTUDIO}

\section{TRIGALES ALTOS}

En el deslizamiento de Trigales Altos algunas infraestructuras se han desplazado más de $20 \mathrm{~cm}$ en el periodo de tiempo analizado en este proyecto, y hasta $4 \mathrm{~m}$ desde la fecha de construcción de la urbanización (Figura 7 a Figura 9). En la Figura 9 se representan las posibles superficies de deslizamiento. La profundidad de la superficie de rotura principal se ha obtenido a partir de los datos de las tomografías sísmicas realizadas.También se realizó una tomografía de resistividad eléctirica (ERT) según el perfil longitudinal del deslizamiento, pero debido a un fallo en uno de los cables del equipo, los resultados no fueron fiables y no se presentan en este informe. Todo este trabajo ha servido para la realización de una tesis de grado del estudiante Irwin Rashid Samaniego Placencia, la cual será defendida en abril de 2018.

Además se ha realizado un vuelo con drone de todo el deslizamiento. En la Figura 10 se puede observar la nube de puntos.

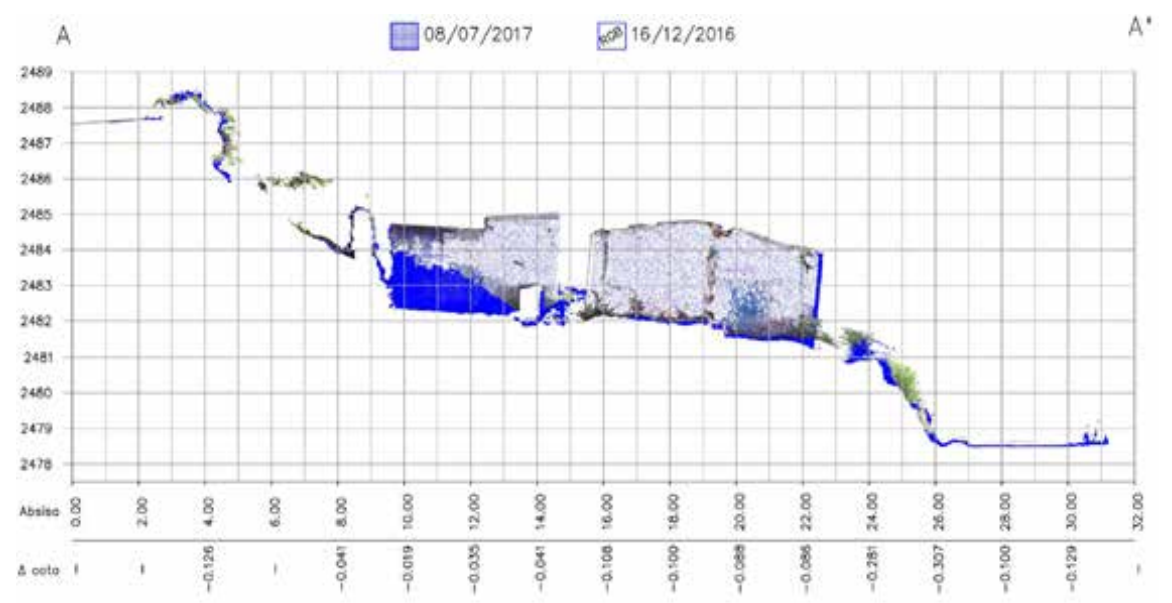

Figura 7. Perfil A-A': levantamiento 1 (RGB) Vs levantamiento 3 (azul).

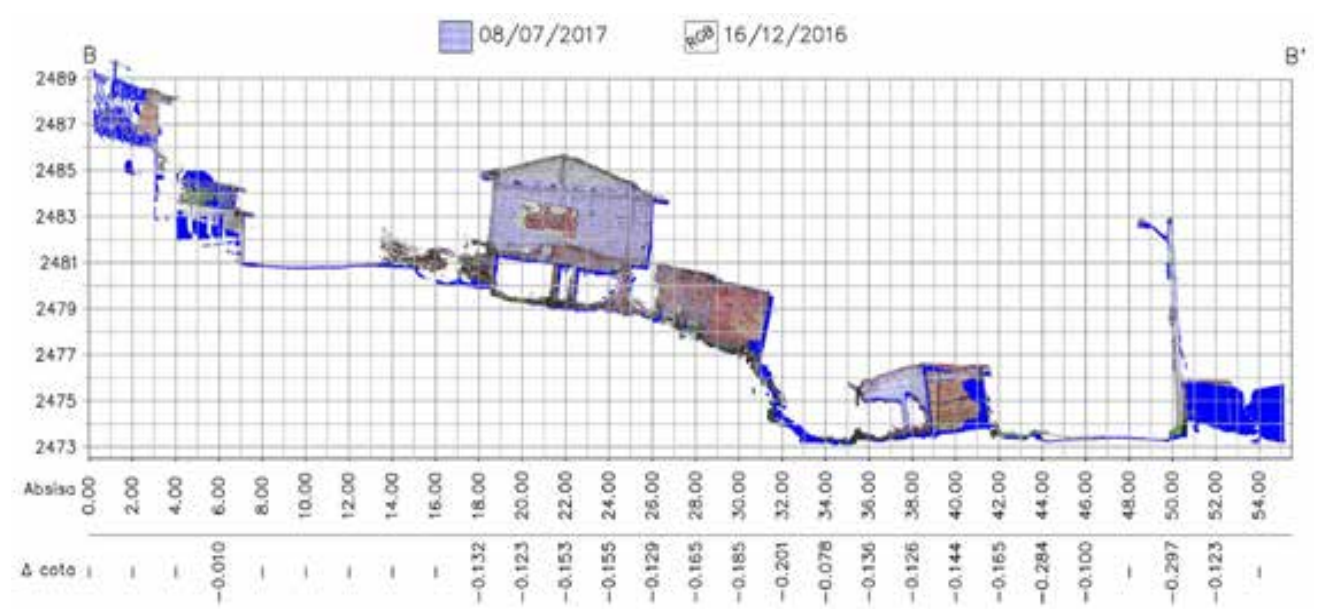

Figura 8. Perfil B-B': levantamiento 1 (RGB) Vs levantamiento 3 (azul). 


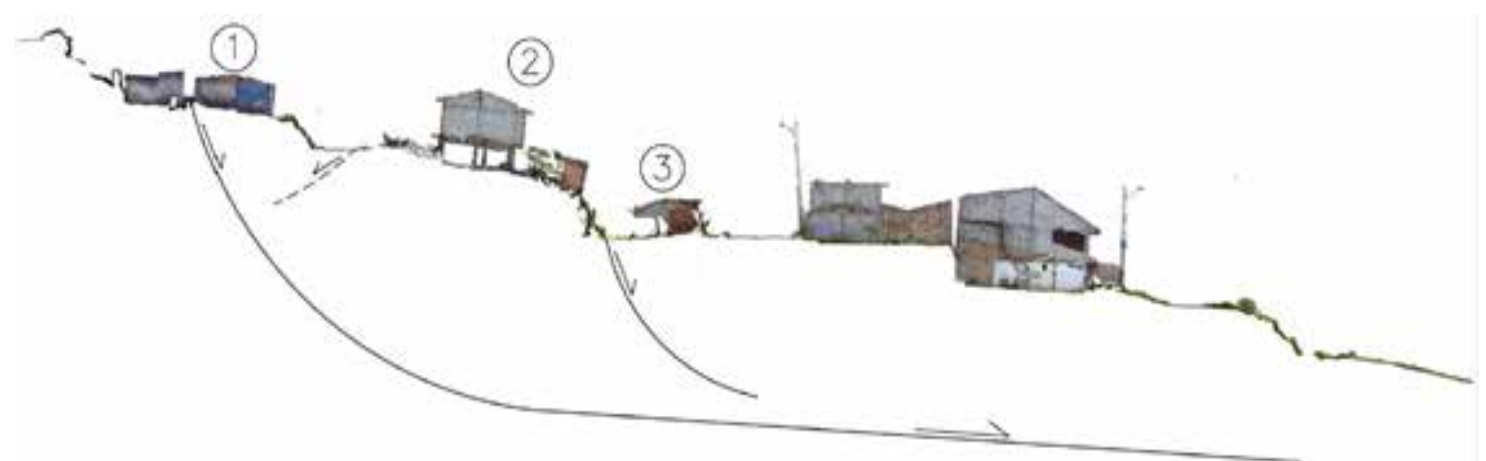

Figura 9. Esquema de posibles superficies de deslizamiento a lo largo del perfil longitudinal de la urbanización Trigales Altos.

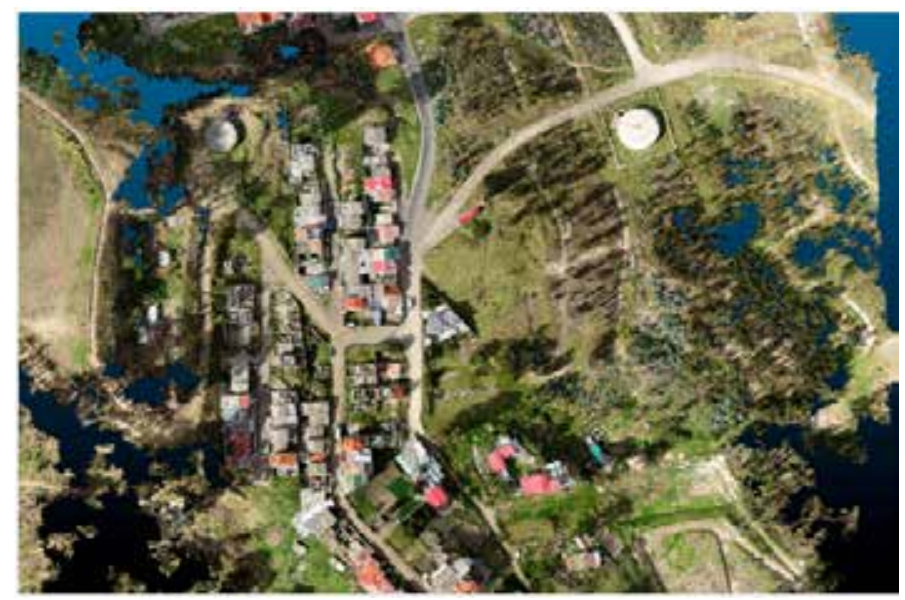

Figura 10. Nube de puntos obtenida mediante fotogrametría drone en Trigales Altos.

\section{REINA DEL CISNE}

En Reina del Cisne el deslizamiento ha sido evidentemente activo durante el periodo de tiempo analizado. Como se aprecia en la Figura 11, el escarpe principal ha aumentado su altura a mayor velocidad durante los meses de lluvia (marzo a mayo). Ladera abajo, esto se ha traducido en importantes deformaciones en una vivienda, con desplazamientos de hasta $2.28 \mathrm{~m}$ en un fragmento de losa. Los desplazamientos de la masa deslizada medidos con LiDAR en los puntos de control coinciden con los obtenidos con la estación total. Este trabajo ha sido recientemente publicado:

Acero P, Caldas M, Mejía P, Pesántez C, Piedra R, Morocho C y Acosta E. (2017). Tipología y cinética del deslizamiento Reina del Cisne (Cuenca) a partir de fotografías aéreas, GPS, escáner láser terrestre y ensayos geotécnicos del suelo. Geoespacial Vol. 14 $\mathrm{N}^{\mathrm{0}} 2.50-66$.

También se ha realizado un vuelo con drone de todo el deslizamiento. En la Figura 12 se puede observar la nube de puntos obtenida con Agisoft Photoscan. 


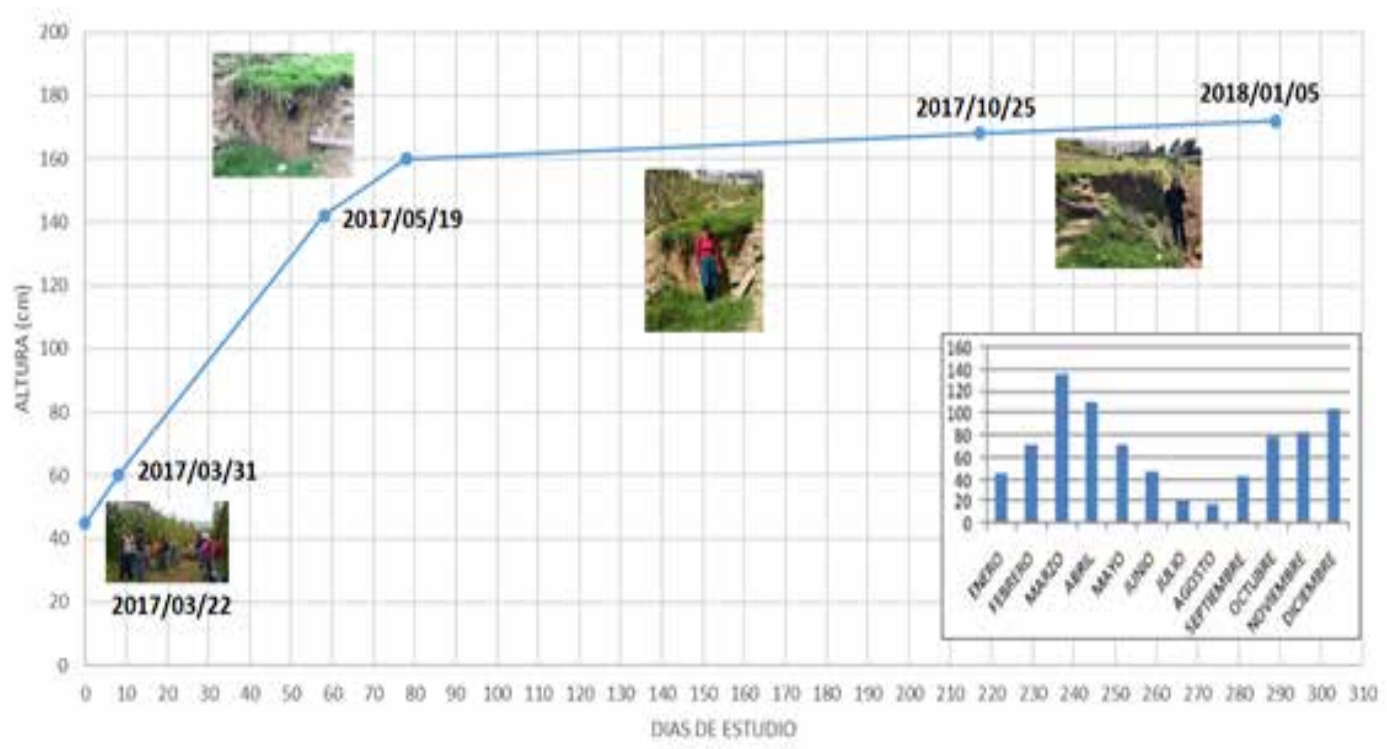

Figura 11. Aumento de altura del escarpe principal del deslizamiento.



Figura 12. Nube de puntos obtenida mediante fotogrametría drone en Reina del Cisne.

\section{SECTOR GAPAL-UNIVERSIDAD DEL AZUAY}

En el deslizamiento de la UDA todavía no se tienen aún resultados definitivos porque sólo se ha realizado un levantamiento drone de toda la zona (Figura 13) y un levantamiento TLS (Figura 14) de algunas infraestructuras situadas en el eje longitudinal del deslizamiento. Es necesario dejar pasar unos meses, especialmente los de lluvia, para que el movimiento se reactive y pueda ser detectado por los equipos. 


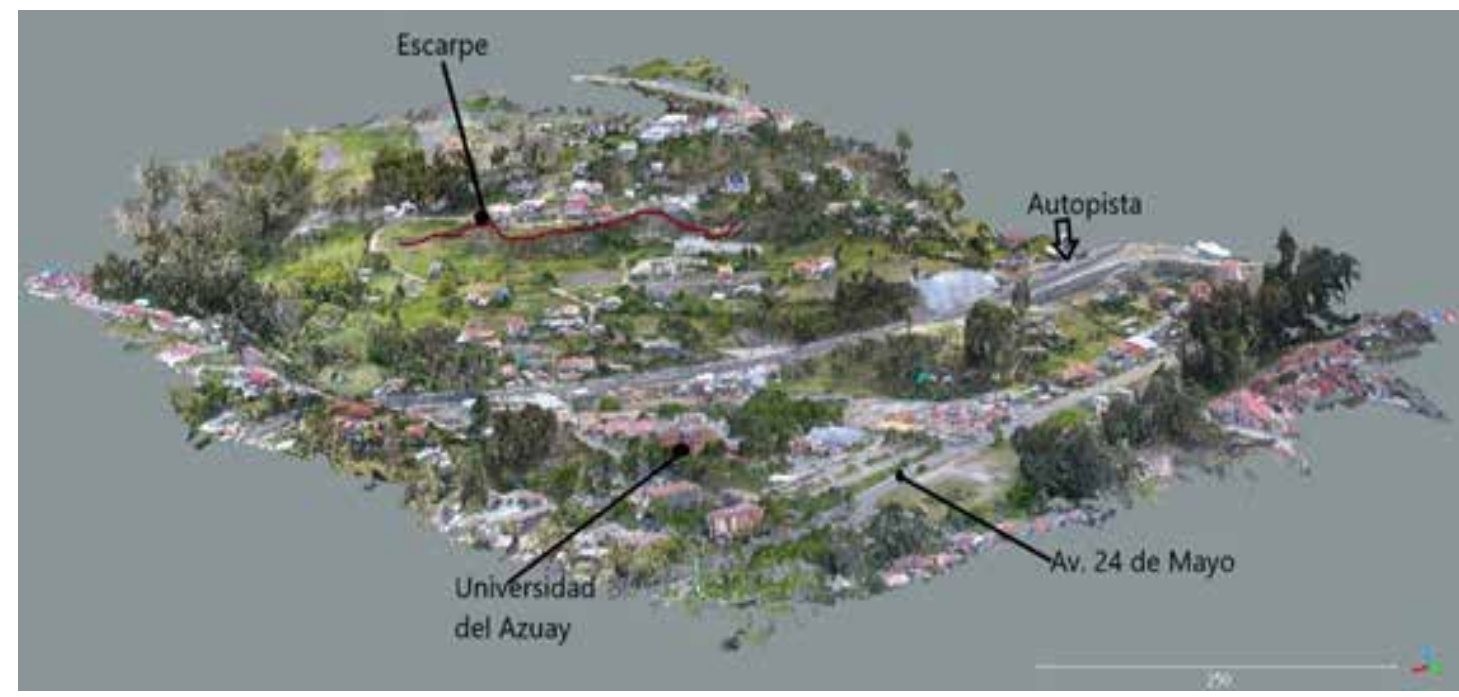

Figura 13. Nube de puntos del deslizamiento UDA obtenida mediante fotogrametría drone.

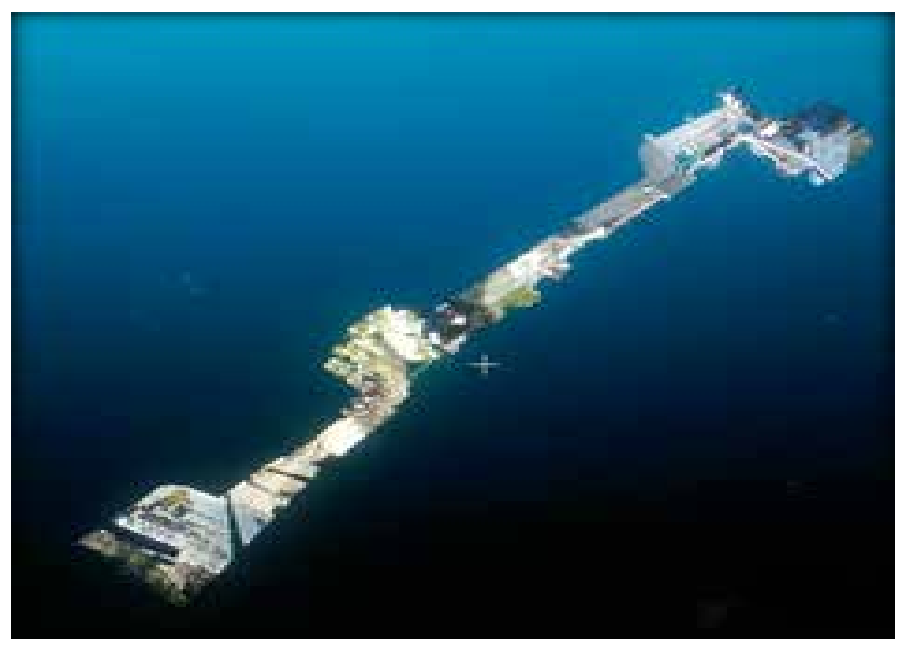

Figura 14. Nube de puntos TLS a lo largo del eje longitudinal del deslizamiento UDA.

\section{COMPARACIÓN DE LOS RESULTADOS DE LAS ZONAS DE ESTUDIO}

Para la comparación de resultados se ha tomado como capas base la cartografía del Proyecto PRECUPA 2011, el servicio WMS de la susceptibilidad de movimientos en masa de la Secretaría Nacional de Gestión de Riesgos y los datos registrados a través de la aplicación móvil.

Según estos datos se puede constatar que el uso de la aplicación móvil ayuda en gran medida al mapeo de datos respecto a las zonas afectadas por deslizamientos y que así se puede reportar nuevos fenómenos o a su vez mantener actualizada la cartografía. 


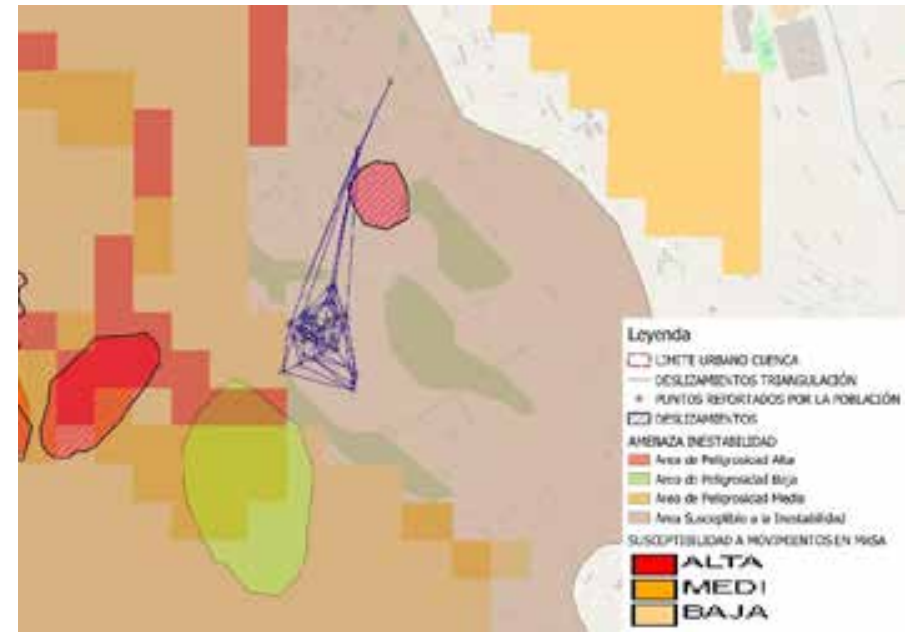

Figura 15. Comparación de cartografía y datos levantados por la población, sector Trigales Altos

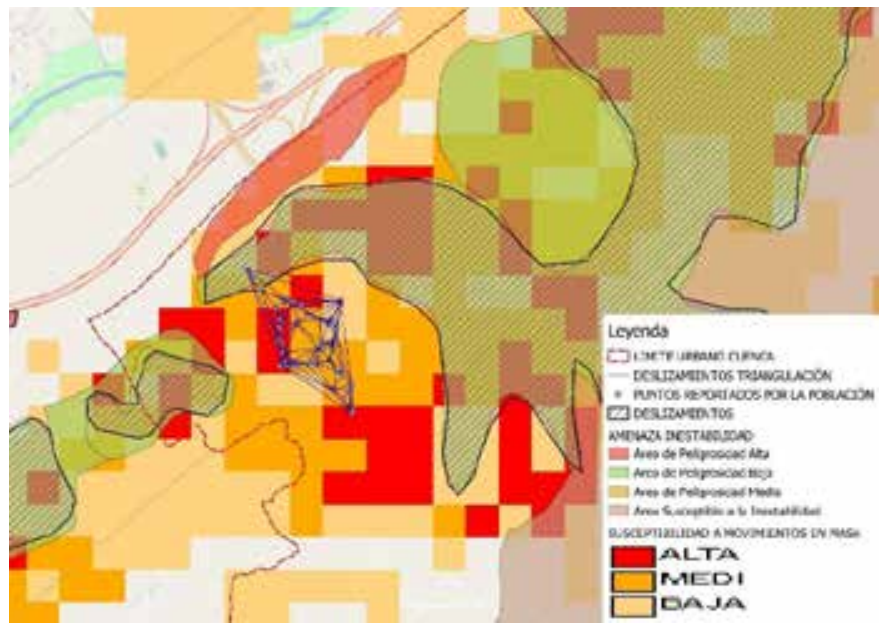

Figura 16. Comparación de cartografia y datos levantado por la población, sector Reina del Cisne

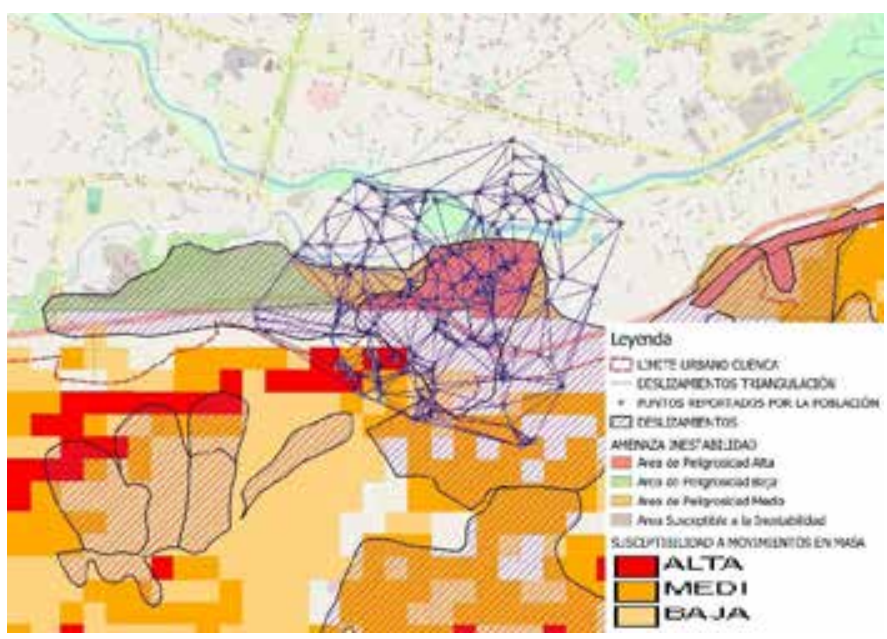

Figura 17. Comparación de cartografía y datos levantado por la población, sector Gapal-UDA 
Es importante recalcar que los puntos que reporta la población no son del todo precisos, sin embargo, pueden ayudar a los tomadores de decisiones y técnicos a contar con un insumo de fácil acceso, de primera mano y actualizado.

Esta potencialidad podrá ser aprovechada, sobretodo, por aquellos GAD que no disponen de recursos económicos ni el contingente para mantener actualizada la cartografía de vulnerabilidades.

\section{CONCLUSIONES}

Los PPGIS son una herramienta que ofrece un enfoque alternativo para la participación del público en la toma de decisiones, permitiendo incorporar los conocimientos locales, integrar y contextualizar la información espacial compleja. En este caso específico se ha implementado una aplicación web móvil que permite al ciudadano identificar las zonas vulnerables, de acuerdo a parámetros establecidos de fácil entendimiento para cualquier usuario.

Las técnicas de teledetección también permiten analizar y actualizar los datos geoespaciales para contar con cartografía más actulizada y que sirven de insumo para la planificación territorial.

\section{AGRADECIMIENTOS}

Este proyecto ha sido financiado por la Dirección de Investigación de la Universidad de Cuenca (DIUC). Agradecimientos al grupo de trabajo de IDE del Departamento de Ciencias de la Computación.

\section{REFERENCIAS}

Acero P, Caldas M, Mejía P, Pesántez C, Piedra R, Morocho C y Acosta E. (2017). Tipología y cinética del deslizamiento Reina del Cisne (Cuenca) a partir de fotografías aéreas, GPS, escáner láser terrestre y ensayos geotécnicos del suelo. Geoespacial Vol. $14 \mathrm{~N}^{\circ}$ 2. 50-66.

Brown, G. (2012). Public Participation GIS (PPGIS) for Regional and Environmental Planning: Reflections on a Decade of Empirical Research, 25(2).

CONSULSUA. (2013). Estudio de impacto ambiental definitivo (eiad) sistema de transmisión de extra alta tensión y sistemas asociados. Recuperado a partir de file://C:/Users/ USUARIO/AppData/Roaming/Mozilla/Firefox/Profiles/trbao6iw.default/zotero/storage/ CQVRAA9P/An\%C3\%A1lisis\%20de\%20Riesgos.pdf

Costa, J. C., López, L., \& Taberner, J. (2000). Epistemological pluralism, participative science and knowledge dialogue as cultural renovation methods. Cultura y Educación, 12(1-2), 181-187. https://doi.org/10.1174/113564000753837287

Morocho, V. (2013). Aportes a la lectura de la vulnerabilidad, a partir de los resultados de los análisis realizados a nivel cantonal. Recuperado a partir de http://repositorio.cedia.org.ec/ handle/123456789/866 